\title{
Fluctuations in the Canonical Ensemble
}

\author{
V.V. Begun ${ }^{a}$, M.I. Gorenstein ${ }^{a, b}$, O.S. Zozulya ${ }^{a, c}$ \\ a Bogolyubov Institute for Theoretical Physics, Kiev, Ukraine \\ ${ }^{b}$ Frankfurt Institute for Advanced Studies, Frankfurt, Germany \\ c Utrecht University, Utrecht, The Netherlands
}

\begin{abstract}
The particle number and energy fluctuations in the system of charged particles are studied in the canonical ensemble for non-zero net values of the conserved charge. In the thermodynamic limit the fluctuations in the canonical ensemble are different from the fluctuations in the grand canonical one. The system with several species of particles is considered. We calculate the quantum statistics effects which can be taken into account for the canonical ensemble fluctuations in the infinite volume limit. The fluctuations of the particle numbers in the pion-nucleon gas are considered in the canonical ensemble as an example of the system with two conserved charges - baryonic number and electric charge.
\end{abstract}

\section{INTRODUCTION}

The statistical model approach turns out to be rather successful in describing the data on the particle production in relativistic nuclear collisions (see e.g. Ref. [1] and recent review [2]). This stimulates further investigation of the properties of the statistical models. In particular, an applicability of various statistical ensembles is an interesting issue. The canonical ensemble (CE) [3] or even the microcanonical ensemble (MCE) [4] have been used in order to describe the $p p, p \bar{p}$ and $e^{+} e^{-}$collisions when a small number of secondary particles are produced. At these conditions the statistical systems are far away from the thermodynamic limit, so that the statistical ensembles are not equivalent, and the exact charge or both energy and charge conservation laws have to be taken into account. The grand canonical ensemble (GCE) formulation is valid when the system volume $V$ tends to infinity. All statistical ensemble become then thermodynamically equivalent.

The analysis of the fluctuations is a useful tool to study the properties of the system created during high energy particle and nuclear collisions (see e.g. Refs. 5, 6, 7] ). An essential part of the total fluctuations measured on the event-by-event basis is expected to be the thermal ones. The particle number fluctuations have been recently studied in the CE [8] and MCE [9] and compared with those in the GCE. It has been shown that these fluctuations are different in various statistical ensembles in the particular case of the relativistic ideal gas with a total net charge equal zero in the Boltzmann statistics approximation. The fluctuations of negatively and positively charged particles are suppressed in the CE [8] in comparison to the fluctuations in the GCE. This suppression remains valid in the thermodynamic limit too, so that the well-known equivalence of all statistical ensembles refers to the average quantities, but does not apply to the fluctuations.

In Ref. 8] we have studied the $\mathrm{CE}$ for one particle specie and zero net value of the conserved charge. In the present paper we extend our consideration. In the high energy proton-proton and nucleus-nucleus collisions the created system has some positive values of the baryonic number and electric charge. Besides, a lot of different species of hadrons are created. We study the CE particle number fluctuations (Secs. II and III) and energy fluctuations (Sec. IV) in the systems with non-zero net charge and several species of particles. As the electric charge of hadrons can be both \pm 1 and \pm 2 , we consider the CE system of single and double charged particles in Sec V. The effects of Bose and Fermi statistics are studied in the thermodynamic limit in Sec. VI. We also calculate in Sec. VII the CE particle number fluctuations for the ideal pion-nucleon gas which is an example of the system with two conserved charges - baryonic number and electric charge. We summarize our consideration and formulate the conclusions in Sec. VIII.

\section{THE GCE AND CE PARTITION FUNCTIONS AND MEAN PARTICLE MULTIPLICITIES}

Let us start with the multi-species system of +1 and -1 charged particles. In applications of the statistical approach to hadron production in high energy collisions the conserved charge under consideration can be the electric charge and baryonic number, or strangeness and charm, which are also conserved in the strong interactions. In the case of the Boltzmann ideal gas (i.e. the interactions and quantum statistics effects are neglected) the partition function in the GCE reads: 


$$
\begin{gathered}
Z_{\text {g.c.e. }}(V, T, \mu)=\sum_{N_{1+}, N_{1-}=0}^{\infty} \ldots \sum_{N_{j+}, N_{j-}=0}^{\infty} \ldots \frac{\left(\lambda_{1+} z_{1}\right)^{N_{1+}}}{N_{1+} !} \frac{\left(\lambda_{1-} z_{1}\right)^{N_{1-}}}{N_{1-} !} \ldots \frac{\left(\lambda_{j+} z_{j}\right)^{N_{j+}}}{N_{j+} !} \frac{\left(\lambda_{j-} z_{j}\right)^{N_{j-}}}{N_{j-} !} \ldots \\
=\prod_{j} \sum_{N_{j+}, N_{j-}=0}^{\infty} \frac{\left(\lambda_{j+} z_{j}\right)^{N_{j+}}}{N_{j+} !} \frac{\left(\lambda_{j-} z_{j}\right)^{N_{j-}}}{N_{j-} !}=\prod_{j} \exp \left(\lambda_{j+} z_{j}+\lambda_{j-} z_{j}\right)=\exp \left[2 z \cosh \left(\frac{\mu}{T}\right)\right],
\end{gathered}
$$

where $j$ numerates the spesies, $\lambda_{j \pm}=\exp ( \pm \mu / T), z_{j}$ is a single particle partition function

$$
z_{j}=\frac{g_{j} V}{2 \pi^{2}} \int_{0}^{\infty} k^{2} d k \exp \left[-\frac{\left(k^{2}+m_{j}^{2}\right)^{1 / 2}}{T}\right]=\frac{g_{j} V}{2 \pi^{2}} T m_{j}^{2} K_{2}\left(\frac{m_{j}}{T}\right),
$$

and $z \equiv \sum_{j} z_{j}$. The $V, T$ and $\mu$ are respectively the system volume, temperature and chemical potential connected with the conserved charge $Q$. The $g_{j}$ and $m_{j}$ are respectively the degeneracy factors and masses for the $j$-th particle species, and $K_{2}$ is the modified Hankel function. The CE partition function is obtained by an explicit introduction of the charge conservation constrain, $\sum_{j}\left(N_{j+}-N_{j-}\right)=Q$, for each microscopic state of the system:

$$
\begin{aligned}
& Z_{c . e .}(V, T, Q)=\sum_{N_{1+}, N_{1-}=0}^{\infty} \ldots \sum_{N_{j+}, N_{j-}=0}^{\infty} \ldots \frac{\left(\lambda_{1+} z_{1}\right)^{N_{1+}}}{N_{1+} !} \frac{\left(\lambda_{1-} z_{1}\right)^{N_{1-}}}{N_{1-} !} \ldots \frac{\left(\lambda_{j+} z_{j}\right)^{N_{j+}}}{N_{j+} !} \frac{\left(\lambda_{j-} z_{j}\right)^{N_{j-}}}{N_{j-} !} \ldots \\
& \times \delta\left[\left(N_{1+}+\ldots+N_{j+}+\ldots-N_{1-}-\ldots-N_{j-}-\ldots\right)-Q\right]=\int_{0}^{2 \pi} \frac{d \phi}{2 \pi} \prod_{j} \sum_{N_{j+}, N_{j-}=0}^{\infty} \frac{\left(\lambda_{j+} z_{j}\right)^{N_{j+}}}{N_{j+} !} \frac{\left(\lambda_{j-} z_{j}\right)^{N_{j-}}}{N_{j-} !} \\
& \times \exp \left[i\left(N_{j+}-N_{j-}-Q\right) \phi\right]=\int_{0}^{2 \pi} \frac{d \phi}{2 \pi} \exp \left[-i Q \phi+\sum_{j} z_{j}\left(\lambda_{j+} e^{i \phi}+\lambda_{j-} e^{-i \phi}\right)\right]=I_{Q}(2 z) .
\end{aligned}
$$

Parameters $\lambda_{j+}$ and $\lambda_{j-}$ in the CE (3) are only auxiliary parameters introduced in order to calculate the mean number and the fluctuations of positively and negatively charged particles. They are set to one in the final formulas. In Eq. (3) the integral representations of the $\delta$-Kronecker symbol and the modified Bessel function were used [10]:

$$
\delta(n)=\int_{0}^{2 \pi} \frac{d \phi}{2 \pi} \exp (i n \phi), \quad I_{Q}(2 z)=\int_{0}^{2 \pi} \frac{d \phi}{2 \pi} \exp [-i Q \phi+2 z \cos \phi] .
$$

The averages of $N_{j+}$ and $N_{j-}$ in both the GCE and CE can be presented as (in the final expressions one should put $\lambda_{j \pm}=\exp ( \pm \mu / T)$ and $\lambda_{j \pm}=1$ for the GCE and CE, respectively):

$$
\left\langle N_{j \pm}\right\rangle=\left(\lambda_{j \pm} \frac{\partial \ln Z}{\partial \lambda_{j \pm}}\right)=a_{ \pm} z_{j}
$$

where $a_{ \pm}$in Eq. (5) is

$$
a_{ \pm}^{\text {g.c.e. }}=\exp \left( \pm \frac{\mu}{T}\right), \quad a_{ \pm}^{\text {c.e. }}=\frac{I_{Q \mp 1}(2 z)}{I_{Q}(2 z)}
$$

for the GCE and CE, respectively. The average number of $N_{+}$and $N_{-}$are equal to:

$$
\left\langle N_{ \pm}\right\rangle=\left\langle\sum_{j} N_{j \pm}\right\rangle=a_{ \pm} \sum_{j} z_{j}=a_{ \pm} z .
$$

The mean net charge in the GCE is equal to:

$$
Q=\left\langle N_{+}\right\rangle_{g . c . e .}-\left\langle N_{-}\right\rangle_{g . c . e .}=2 \sinh \left(\frac{\mu}{T}\right) z .
$$

which leads to a simple relation which connects the values of $Q$ and $\mu$

$$
\exp \left(\frac{\mu}{T}\right)=\frac{Q}{2 z}+\sqrt{1+\left(\frac{Q}{2 z}\right)^{2}} \equiv y+\sqrt{1+y^{2}},
$$


so that

$$
\left\langle N_{ \pm}\right\rangle_{\text {g.c.e. }}=z\left(y+\sqrt{1+y^{2}}\right)^{ \pm 1}
$$

where $y \equiv Q / 2 z=\sinh (\mu / T)$.

In the $\mathrm{CE}$ an exact charge conservation is imposed on each microscopic state, so that it is evidently fulfilled also for the average values:

$$
\left\langle N_{+}\right\rangle_{c . e .}-\left\langle N_{-}\right\rangle_{c . e .}=z \frac{I_{Q-1}(2 z)}{I_{Q}(2 z)}-z \frac{I_{Q+1}(2 z)}{I_{Q}(2 z)}=Q,
$$

as indeed can be easily seen from the identity $I_{n-1}(x)-I_{n+1}(x)=2 n I_{n}(x) / x$ [10].

The ratios of $\left\langle N_{ \pm}\right\rangle$calculated in the CE and in the GCE,

$$
\frac{\left\langle N_{ \pm}\right\rangle_{\text {c.e. }}}{\left\langle N_{ \pm}\right\rangle_{\text {g.c.e. }}}=\frac{I_{Q \mp 1}(2 z)}{I_{Q}(2 z)} \cdot\left(y+\sqrt{1+y^{2}}\right)^{\mp 1}
$$

are shown in Fig. 1 for $Q=0$ and $Q=2$. There is the strong canonical suppression effect, $\left\langle N_{ \pm}\right\rangle_{c . e .} \ll\left\langle N_{ \pm}\right\rangle_{\text {g.c.e. }}$, for small systems $(z \ll 1)$, and the canonical and grand canonical ensembles become equivalent, $\left\langle N_{ \pm}\right\rangle_{\text {c.e. }}=\left\langle N_{ \pm}\right\rangle_{\text {g.c.e. }}$, in the thermodynamic limit $z \rightarrow \infty$. One can see that the $\mathrm{CE}$ suppression effect is reduced for a non-zero net charge of the system as compared to a system with zero net charge. In Fig. 2 the ratios (12) as functions of $Q=1,2, \ldots$ are shown at fixed positive values of $y=Q / 2 z$ which correspond to the fixed positive net charge number densities $(Q=0$ corresponds to $y=0$ and is presented in Fig. 1). Small values of $y$ mean large $z$, e.g. for $y=0.1$ shown in Fig. 2 one finds 'large' $z=5$ at $Q=1$, so that the system is already close to the thermodynamic limit. Due to this the canonical suppression is small and it is the same for positive and negative particles. The case of large $y$ differs, e.g. for $y=2$ shown in Fig. 2 the values of $z$ are 'small' at small $Q: z=0.25$ at $Q=1$. The canonical suppression effect becomes strong for negative particles at small $Q$. However, the canonical suppression at large $y$ is negligible for the average value of positive particle number as it should be approximately equal to $Q$.

For small systems $(z \ll 1)$ using the series expansion $[10$ ]

$$
I_{n}(2 z)=\frac{z^{n}}{n !}+\frac{z^{n+2}}{(n+1) !}+O\left(z^{n+4}\right),
$$

one finds for $Q=0$

$$
\left\langle N_{ \pm}\right\rangle_{\text {c.e. }} \simeq z^{2} \ll\left\langle N_{ \pm}\right\rangle_{\text {g.c.e. }}=z
$$

and for $Q \geq 1$

$$
\begin{aligned}
& \left\langle N_{+}\right\rangle_{\text {c.e. }} \simeq Q, \quad\left\langle N_{+}\right\rangle_{\text {c.e. }} \simeq\left\langle N_{+}\right\rangle_{\text {g.c.e. }} ; \\
& \left\langle N_{-}\right\rangle_{\text {c.e. }} \simeq \frac{z^{2}}{Q+1}, \quad\left\langle N_{-}\right\rangle_{\text {c.e. }} \simeq \frac{Q}{Q+1}\left\langle N_{-}\right\rangle_{\text {g.c.e. }} .
\end{aligned}
$$

In the large volume limit $(V \rightarrow \infty$ corresponds also to $z \rightarrow \infty)$ the mean quantities in the CE and GCE are equal. This result is referred to as an equivalence of the canonical and grand canonical ensembles. Using the uniform limit of the modified Bessel function [10]

$$
\lim _{n \rightarrow \infty} I_{n}(n x)=\frac{1}{\sqrt{2 \pi n}} \frac{\exp (\eta n)}{\left(1+x^{2}\right)^{1 / 4}}\left[1+O\left(\frac{1}{n}\right)\right]
$$

where

$$
\eta=\sqrt{1+x^{2}}+\log \frac{x}{1+\sqrt{1+x^{2}}},
$$

one can easily find (note that fixed $Q$ at $z \rightarrow \infty$ means a zero value of the net charge density and $y=0$ ):

$$
\left\langle N_{ \pm}\right\rangle_{c . e .} \simeq z\left(y+\sqrt{1+y^{2}}\right)^{ \pm 1}=\left\langle N_{ \pm}\right\rangle_{\text {g.c.e. }} .
$$

The total multiplicity of charged particles is defined as $N_{c h}=N_{+}+N_{-}$. Its average in the GCE and CE reads:

$$
\begin{aligned}
\left\langle N_{c h}\right\rangle_{\text {g.c.e. }} & \equiv\left\langle N_{+}+N_{-}\right\rangle_{\text {g.c.e. }}=\left\langle N_{+}\right\rangle_{\text {g.c.e. }}+\left\langle N_{-}\right\rangle_{\text {g.c.e. }}=2 z \cosh \left(\frac{\mu}{T}\right), \\
\left\langle N_{\text {ch }}\right\rangle_{\text {c.e. }} & \equiv\left\langle N_{+}+N_{-}\right\rangle_{\text {c.e. }}=\left\langle N_{+}\right\rangle_{\text {c.e. }}+\left\langle N_{-}\right\rangle_{\text {c.e. }}=z\left[\frac{I_{Q-1}(2 z)}{I_{Q}(2 z)}+\frac{I_{Q+1}(2 z)}{I_{Q}(2 z)}\right] .
\end{aligned}
$$



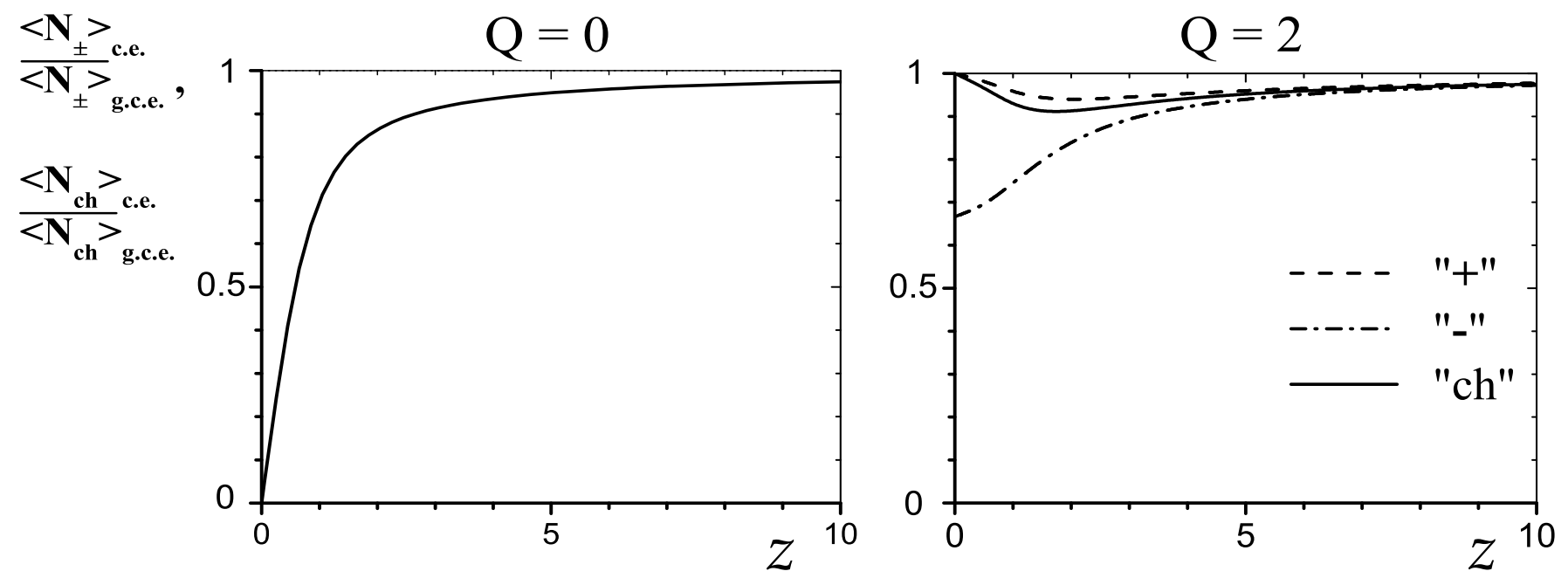

FIG. 1: The ratios of the mean particle numbers in the CE to those in the GCE as functions of $z$ for $Q=0$ and $Q=2$.
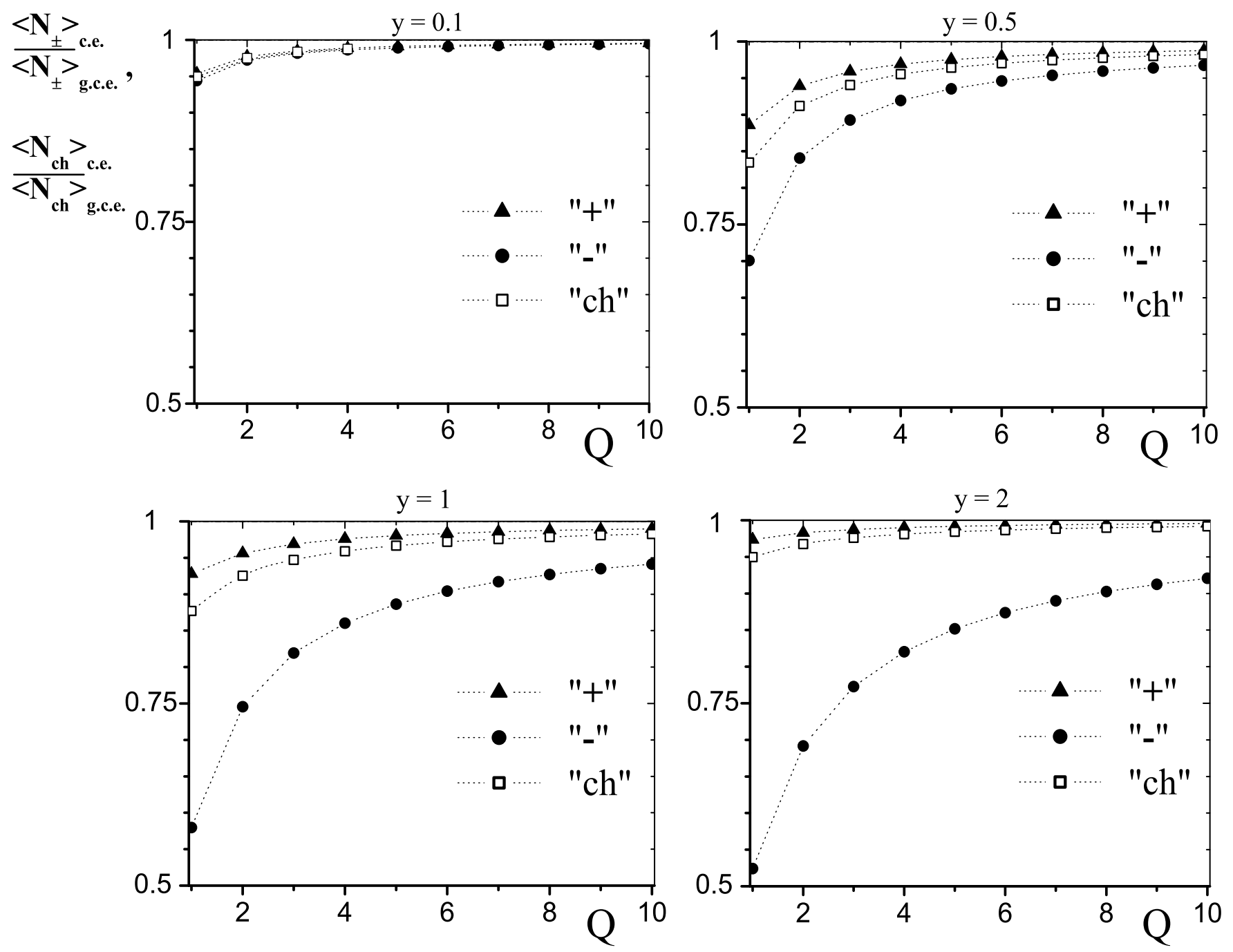

FIG. 2: The ratios of the mean particle numbers in the CE to those in the GCE as functions of $Q=1,2,3, \ldots$ for fixed values of $y=Q / 2 z$. 


\section{THE SCALED VARIANCE}

A useful measure of the fluctuations of any variable $X$ is the ratio of its variance $V(X) \equiv\left\langle X^{2}\right\rangle-\langle X\rangle^{2}$ to its mean value $\langle X\rangle$, referred here as the scaled variance:

$$
\omega^{X} \equiv \frac{\left\langle X^{2}\right\rangle-\langle X\rangle^{2}}{\langle X\rangle}
$$

Note, that $\omega^{X}=1$ for the Poisson distribution. In order to study the fluctuations of charged particle numbers the second moments of the multiplicity distribution have to be calculated. One finds:

$$
\begin{aligned}
\left\langle N_{j \pm}^{2}\right\rangle & =\frac{1}{Z}\left[\lambda_{j \pm} \frac{\partial}{\partial \lambda_{j \pm}}\left(\lambda_{j \pm} \frac{\partial Z}{\partial \lambda_{j \pm}}\right)\right]=a_{ \pm} z_{j}+b_{ \pm} z_{j}^{2}, \\
\left\langle N_{j+} N_{j-}\right\rangle & =\frac{\lambda_{j+} \lambda_{j-}}{Z} \frac{\partial^{2} Z}{\partial \lambda_{j+} \partial \lambda_{j-}}=z_{j}^{2}
\end{aligned}
$$

where $a_{ \pm}$is given by Eq. (6) and

$$
b_{ \pm}^{\text {g.c.e. }}=\exp \left( \pm \frac{2 \mu}{T}\right)=\left(a_{ \pm}^{\text {g.c.e. }}\right)^{2}, \quad b_{ \pm}^{\text {c.e. }}=\frac{I_{Q \mp 2}(2 z)}{I_{Q}(2 z)},
$$

in the GCE and CE, respectively. The scaled variances $\omega^{j \pm}$ and $\omega^{j c h}$ are equal to:

$$
\begin{aligned}
\omega^{j \pm} & \equiv \frac{\left\langle N_{j+}^{2}\right\rangle-\left\langle N_{j+}\right\rangle^{2}}{\left\langle N_{j+}\right\rangle}=1-z_{j}\left(a_{ \pm}-\frac{b_{ \pm}}{a_{ \pm}}\right), \\
\omega^{j c h} & \equiv \frac{\left\langle\left(N_{j+}+N_{j-}\right)^{2}\right\rangle-\left\langle N_{j+}+N_{j-}\right\rangle^{2}}{\left\langle N_{j+}+N_{j-}\right\rangle}=1+z_{j}\left[\frac{b_{+}+b_{-}+2}{a_{+}+a_{-}}-\left(a_{+}+a_{-}\right)\right],
\end{aligned}
$$

The Eqs. (26 27) describe the particle number fluctuations of a given specie $j$. One can establish the general rule how to calculate the fluctuations of $N_{ \pm}=\sum_{j} N_{j \pm}$ and $N_{c h}=N_{+}+N_{-}$. To do this one should set $\lambda_{1 \pm}=\lambda_{2 \pm}=\cdots=$ $\lambda_{ \pm}$in Eqs. (13) and differentiate with respect to $\lambda_{ \pm}$in Eqs. (5123) in order to get $\left\langle N_{ \pm}^{n}\right\rangle(n=1,2)$. This eventually results in a substitution of $z$ instead of $z_{j}$ in all final formulas for the averages and fluctuations. One obtains:

$$
\begin{aligned}
\omega_{\text {g.c.e. }}^{ \pm} & =\omega_{\text {g.c.e. }}^{\text {ch }}=1 . \\
\omega_{\text {c.e. }}^{ \pm} & =1-z\left[\frac{I_{Q \mp 1}(2 z)}{I_{Q}(2 z)}-\frac{I_{Q \mp 2}(2 z)}{I_{Q \mp 1}(2 z)}\right], \\
\omega_{\text {c.e. }}^{\text {ch }} & =1+z\left[\frac{I_{Q-2}(2 z)+I_{Q+2}(2 z)+2 I_{Q}(2 z)}{I_{Q-1}(2 z)+I_{Q+1}(2 z)}-\frac{I_{Q-1}(2 z)+I_{Q+1}(2 z)}{I_{Q}(2 z)}\right] .
\end{aligned}
$$

The scaled variances $\omega_{c . e}^{ \pm}$and $\omega_{c . e}^{c h}$ calculated with Eqs. (29) and (30) are shown in Fig. [3] for $Q=0, Q=2$ and in Fig. [ [ for fixed positive values of $y$. Using the asymptotic behavior of the modified Bessel function for $z \rightarrow 0$, Eq. (13), and $z, Q \rightarrow \infty$ with $y=Q / 2 z=$ const, Eqs. (17.18), the limits of the scaled variances can be easily found, both for a given particle specie $j$ and for the sum over all particle species:

1). A small system limit $z \rightarrow 0$ gives for $Q=0$

$$
\begin{array}{ll}
\omega_{c . e .}^{j+}=\omega_{c . e .}^{-} \simeq 1-\frac{z_{j} z}{2}, & \omega_{c . e .}^{+}=\omega_{c . e .}^{-} \simeq 1-\frac{z^{2}}{2}, \\
\omega_{c . e .}^{j c h} \simeq 1+\frac{z_{j}}{z}-z_{j} z, & \omega_{c . e .}^{c h} \simeq 2-z^{2},
\end{array}
$$

and for $Q \geq 1$

$$
\begin{array}{ll}
\omega_{\text {c.e. }}^{j+} \cong 1-\frac{z_{j}}{z}+\frac{z_{j} z}{Q(Q+1)}, & \omega_{c . e .}^{+} \cong \frac{z^{2}}{Q(Q+1)}, \\
\omega_{c . e .}^{j-} \cong 1-\frac{z_{j} z}{(Q+1)(Q+2)}, & \omega_{c . e .}^{-} \cong 1-\frac{z^{2}}{(Q+1)(Q+2)}, \\
\omega_{c . e .}^{j c h} \cong 1-\frac{z_{j}}{z}+\frac{4 z_{j} z}{Q(Q+1)}, & \omega_{c . e .}^{c h} \cong \frac{4 z^{2}}{Q(Q+1)} .
\end{array}
$$



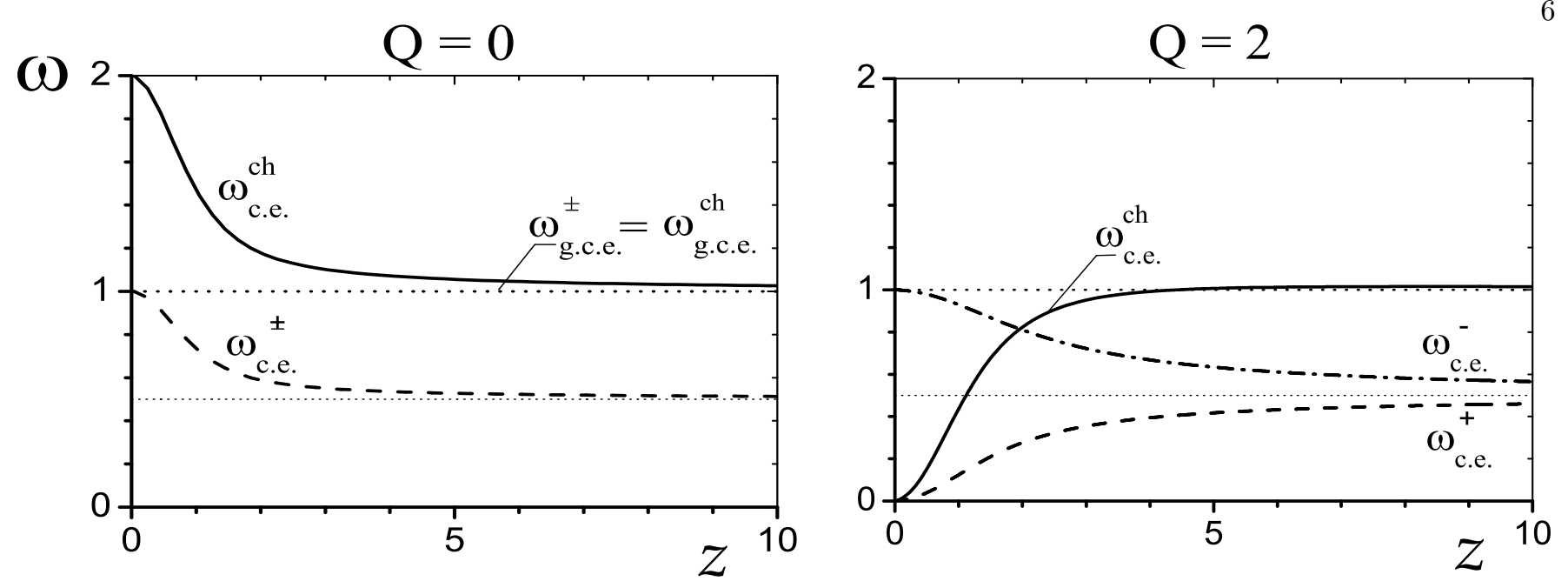

FIG. 3: The scaled variances $\omega_{\text {c.e. }}^{ \pm}$(29) and $\omega_{\text {c.e. }}^{\text {ch }}$ (30) as functions of $z$ for fixed values of the conserved charge $Q$.
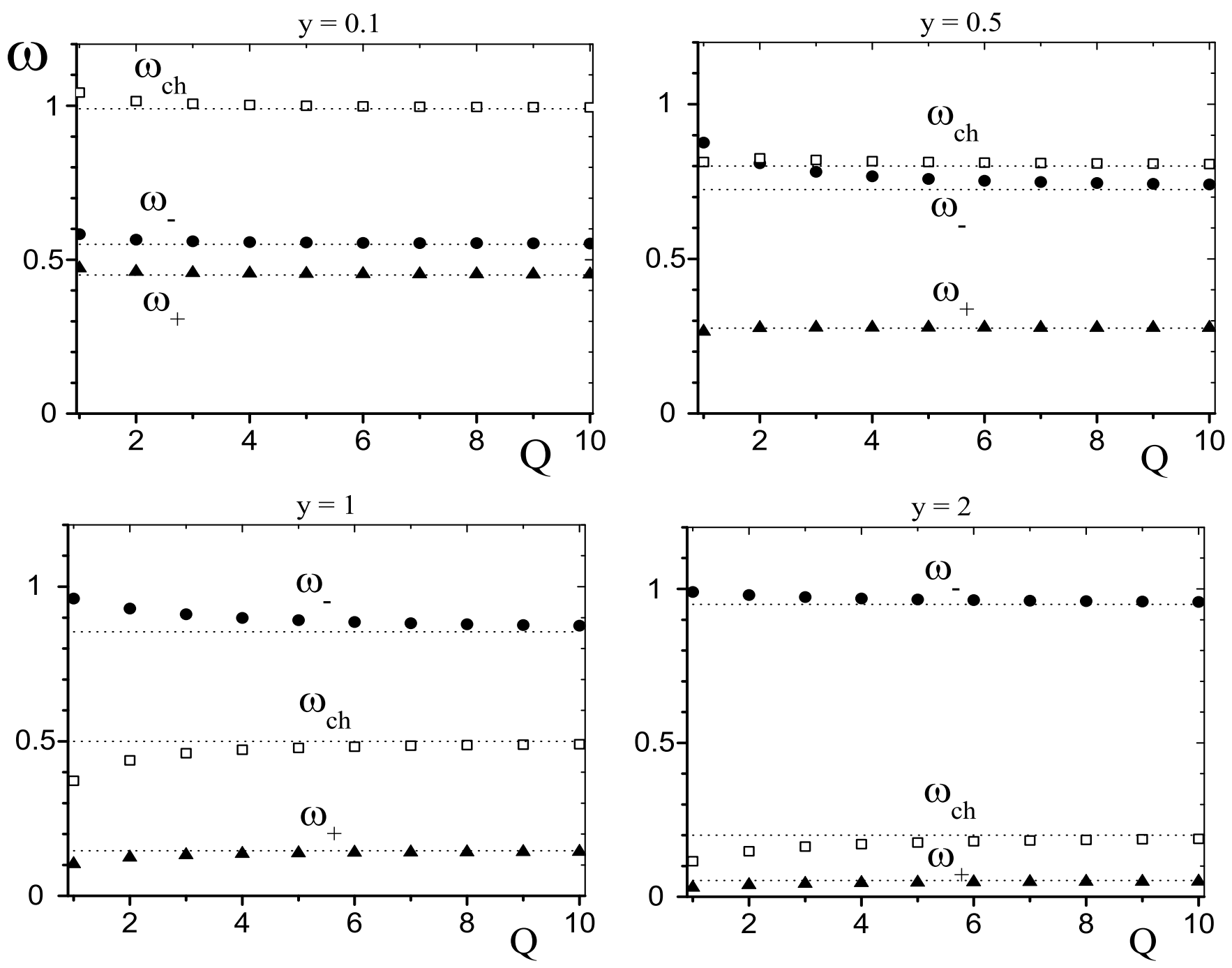

FIG. 4: The scaled variances $\omega_{\text {c.e. }}^{ \pm}$[29) and $\omega_{\text {c.e. }}^{\text {ch }}$ (30) as functions of $Q=1,2,3, \ldots$ for fixed values of $y=Q / 2 z$. 
2). A large system limit $z \rightarrow \infty$ gives for fixed $Q$ (note again that fixed $Q$ in the thermodynamic limit $z \rightarrow \infty$ means a zero value of the net charge density and leads, therefore, to $y=0$ )

$$
\begin{array}{rlrl}
\omega_{c . e .}^{j \pm} & \simeq 1-\frac{z_{j}}{2 z}+\frac{z_{j}}{8 z^{2}} \mp \frac{Q z_{j}}{4 z^{2}}, & \omega_{c . e .}^{ \pm} & \simeq \frac{1}{2}+\frac{1}{8 z} \mp \frac{Q}{4 z}, \\
\omega_{\text {c.e. }}^{j c h} \simeq 1+\frac{z_{j}}{4 z^{2}}, & \omega_{c . e .}^{\text {ch }} & \simeq 1+\frac{1}{4 z},
\end{array}
$$

and for fixed $Q / 2 z=y$

$$
\begin{array}{lll}
\omega_{\text {c.e. }}^{j \pm} \simeq 1-\frac{z_{j}}{2 z} \mp \frac{z_{j}}{2 z} \frac{y}{\sqrt{1+y^{2}}}, & \omega_{\text {c.e. }}^{ \pm} \simeq \frac{1}{2} \mp \frac{y}{2 \sqrt{1+y^{2}}}, \\
\omega_{\text {c.e. }}^{j c h} \simeq 1-\frac{z_{j}}{z} \frac{y^{2}}{1+y^{2}}, & \omega_{\text {c.e. }}^{\text {ch }} \simeq \frac{1}{1+y^{2}} .
\end{array}
$$

As one sees from Eqs. 3-4 the scaled variances reach very fast their asymptotic values. In Fig. [3] the scaled variances for $Q=0$ and $Q=2$ can be compared (for $Q=0$ see details in Ref. [ 8$]$ ). One notices that their values at $z \rightarrow \infty$ are the same, but the behavior at small values of $z$ is different. Namely, if $Q \geq 1$ the fluctuations of positively charged particles are very small at small $z$, while the fluctuations of the negatively charged particles have the Poisson width. This can be easy understood as for small volumes the average number of positive particles is approximately equal to $Q$ (see Eq. (15D) and the fluctuations of $N_{+}$are small. On the other hand, at small $z$ and fixed $Q$ the average number of negatively charged particles is much smaller than $Q$ (see Eq. (16)) and the fluctuations of $N_{-}$are not affected by the conservation law. Similar physical reasons explain the behavior of the fluctuations at non-zero charge density in the thermodynamic limit. The Fig. 4 demonstrates the following features of the asymptotic values of $\omega_{c . e .}^{+}$and $\omega_{c . e}^{-}$. at $Q \gg 1$. When the charge density becomes larger ( $y$ increases) the $\omega_{c . e .}^{+}$decreases and tends to 0 at $y \rightarrow \infty$, while the $\omega_{\text {c.e. }}^{-}$increases and tends to 1 at $y \rightarrow \infty$. The physical reasons of this are seen from Eq. (19) which at $y \gg 1$ gives: $\left\langle N_{+}\right\rangle_{c . e .} \simeq z \cdot 2 y=Q$ and $\left\langle N_{-}\right\rangle_{\text {c.e. }} \simeq z \cdot(2 y)^{-1}=Q \cdot\left(4 y^{2}\right)^{-1} \ll Q$. Therefore, at $y \gg 1$ an exact charge conservation keeps $N_{+}$close to its average value $Q$ and makes the fluctuations of $N_{+}$in the CE to be small. Under the same conditions, $\left\langle N_{-}\right\rangle_{\text {c.e. }}$ is much smaller than $Q$, so that the fluctuations of $N_{-}$are not affected by the CE suppression effects and has the Poisson form. These features of the CE are in a striking difference with those in the GCE. The GCE scaled variances (28) are equal to 1 for $N_{-}, N_{+}$and $N_{c h}$, and this remains valid for all values of the system net charge or net charge density.

\section{ENERGY FLUCTUATIONS}

The partition function in the GCE and $\mathrm{CE}$ is equal to $Z \equiv \sum \exp (-E / T)$, where the sum over microstates includes the summation (integration) over particle momenta and summation over number of particles and over different particle species. Each microscopic state has the weight factor $\prod_{j} \exp \left[\left(\mu N_{j+}-\mu N_{j-}\right) / T\right]$ in the GCE (1)and $\delta\left[Q-\sum_{j}\left(N_{j+}-\right.\right.$ $\left.N_{j-}\right)$ ] in the CE (3). In order to calculate the average energy and its fluctuations it is convenient to rewrite the partition function as $Z=\sum \exp \left[\sum_{j}\left(\beta_{j+} E_{j+}+\beta_{j-} E_{j-}\right) / T\right]$, where $\beta_{j+}$ and $\beta_{j-}$ are the auxiliary parameters and $\beta_{j+}=\beta_{j-}=\beta \equiv 1 / T$ in the final formulas. It then follows:

$$
\begin{aligned}
\left\langle E_{j \pm}\right\rangle & =-\frac{1}{Z} \frac{\partial Z}{\partial \beta_{j \pm}}=-a_{ \pm} z_{j}^{\prime} \equiv\left\langle\varepsilon_{j}\right\rangle\left\langle N_{j \pm}\right\rangle, \\
\left\langle E_{i \pm} E_{j \pm}\right\rangle & =\frac{1}{Z} \frac{\partial^{2} Z}{\partial \beta_{i \pm} \beta_{j \pm}}=a_{ \pm} z_{j}^{\prime \prime} \delta_{i j}+b_{ \pm} z_{i}^{\prime} z_{j}^{\prime}, \\
\left\langle E_{i+} E_{j-}\right\rangle & =\frac{1}{Z} \frac{\partial^{2} Z}{\partial \beta_{i+} \beta_{j-}}=z_{i}^{\prime} z_{j}^{\prime},
\end{aligned}
$$

where $z_{j}^{\prime}=\partial z_{j} / \partial \beta, z_{j}^{\prime \prime}=\partial^{2} z_{j} / \partial \beta^{2}$, and $z_{j}, a_{ \pm}, b_{ \pm}$are given by Eqs. (26]25), respectively. In Eq. (40) we have introduced the average value of one-particle energy $\left\langle\varepsilon_{j}\right\rangle \equiv-z_{j}^{\prime} / z_{j}$. Introducing also $\left\langle\varepsilon_{j}^{2}\right\rangle \equiv z_{j}^{\prime \prime} / z_{j}$ the energy fluctuations can be then presented as:

$$
W^{j \pm} \equiv \frac{\left\langle E_{j \pm}^{2}\right\rangle-\left\langle E_{j \pm}\right\rangle^{2}}{\left\langle E_{j \pm}\right\rangle}=\frac{\left\langle\varepsilon_{j}^{2}\right\rangle-\left\langle\varepsilon_{j}\right\rangle^{2}}{\left\langle\varepsilon_{j}\right\rangle}+\left\langle\varepsilon_{j}\right\rangle \omega^{j \pm},
$$


where $\omega^{j \pm}$ is given by Eq. (26). Introducing the total energies $E_{ \pm} \equiv \sum_{j} E_{j \pm}$ and $E_{c h} \equiv \sum_{j}\left(E_{j+}+E_{j-}\right)$, one finds:

$$
\begin{gathered}
W^{ \pm} \equiv \frac{\left\langle E_{ \pm}^{2}\right\rangle-\left\langle E_{ \pm}\right\rangle^{2}}{\left\langle E_{ \pm}\right\rangle}=\frac{\left\langle\varepsilon^{2}\right\rangle-\langle\varepsilon\rangle^{2}}{\langle\varepsilon\rangle}+\langle\varepsilon\rangle \omega^{ \pm}, \\
W^{c h} \equiv \frac{\left\langle E_{c h}^{2}\right\rangle-\left\langle E_{c h}\right\rangle^{2}}{\left\langle E_{c h}\right\rangle}=\frac{\left\langle\varepsilon^{2}\right\rangle-\langle\varepsilon\rangle^{2}}{\langle\varepsilon\rangle}+\langle\varepsilon\rangle \omega^{c h},
\end{gathered}
$$

where $\langle\varepsilon\rangle \equiv \sum_{j} z_{j}\left\langle\varepsilon_{j}\right\rangle / z,\left\langle\varepsilon^{2}\right\rangle \equiv \sum_{j}\left\langle\varepsilon_{j}^{2}\right\rangle z_{j} / z$. The Eqs. 4445) are valid in both the GCE and CE. The energy fluctuations consist of two terms. The first term takes into account the fluctuations of one-particle energies, the second one - the fluctuations of the number of particles. Most often the fluctuations of the number of particles are relatively more important than the fluctuations of one-particle energies. Indeed, the maximal value of the first term in the right hand side of Eqs. (4445) is equal to $\langle\varepsilon\rangle / 3$ for the particles with $m / T \rightarrow 0$, and it decreases and goes to zero at $m / T \rightarrow \infty$. On the other hand, the second term in the right hand side of Eqs. (44.45) is equal to $\langle\varepsilon\rangle$ for any system in the GCE. The value of $\left(\left\langle\varepsilon^{2}\right\rangle-\langle\varepsilon\rangle^{2}\right) /\langle\varepsilon\rangle$ in Eqs. (4445) is the same for "+" and "-" particles, and in both the GCE and CE. The values of $\omega$ 's are however different in the GCE and CE. Besides, the $\omega_{c . e .}^{+}, \omega_{c . e}^{-}$and $\omega_{c . e \text { e. }}^{\text {ch }}$ are different from each other for the non-zero net charge $Q$. Therefore, the scaled variances of the energy fluctuations are different in the GCE and CE, and in the CE the values of $W^{+}, W^{-}$and $W^{c h}$ differ from each other and depend on the net charge of the system. An example of the energy fluctuations $W^{+}, W^{-}$and $W^{c h}$ for the ideal pion gas with $Q=0$ and $Q=2$ is presented in Fig. One sees that the dependences of the energy fluctuations $W^{+}, W^{-}$and $W^{c h}$ on $z$ in the $\mathrm{CE}$ resemble those for $\omega_{c . e .}^{+}, \omega_{c . e .}^{-}$and $\omega_{c . e .}^{c h}$ shown in Fig. 3
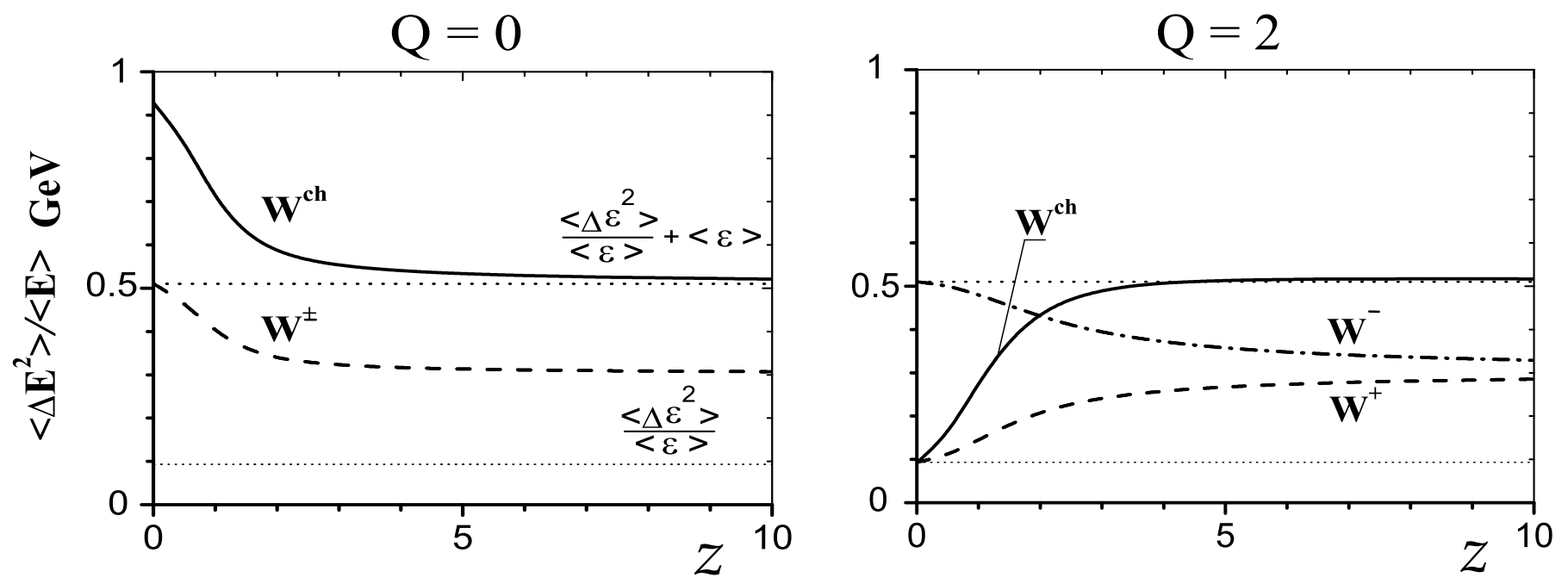

FIG. 5: The CE energy fluctuation $W^{+}, W^{-}$and $W^{c h}$ in the ideal pion gas at temperature $T=120 \mathrm{MeV}$.

The upper horizontal dotted line in Fig. 5 shows the value of $\left\langle\varepsilon^{2}\right\rangle /\langle\varepsilon\rangle$ which corresponds to the $W^{+}=W^{-}=W^{c h}$ in the GCE. The lower horizontal dotted line in Fig. 5 shows the value of $\left(\left\langle\varepsilon^{2}\right\rangle-\langle\varepsilon\rangle^{2}\right) /\langle\varepsilon\rangle$ which is the fluctuations of one-pion energy.

\section{SINGLE AND DOUBLE CHARGED PARTICLES}

In the following sections we consider the extension of the CE formalism. First, let us study the system of particles and antiparticles with charges \pm 1 and \pm 2 . The CE partition function reads:

$$
\begin{aligned}
& Z_{\text {c.e. }}(V, T, Q)=\sum_{N_{+}, N_{-}, \widetilde{N}_{+}, \widetilde{N}_{-}=0}^{\infty} \frac{\left(\lambda_{+} z\right)^{N_{+}}}{N_{+} !} \frac{\left(\lambda_{-} z\right)^{N_{-}}}{N_{-} !} \frac{\left(\widetilde{\lambda}_{+} \widetilde{z}\right)^{\tilde{N}_{+}}}{\widetilde{N}_{+} !} \frac{\left(\widetilde{\lambda}_{-} \widetilde{z}^{\tilde{N}_{-}}\right.}{\widetilde{N}_{-} !} \delta\left[\left(N_{+}-N_{-}+2 \widetilde{N}_{+}-2 \widetilde{N}_{-}\right)-Q\right] \\
& =\int_{0}^{2 \pi} \frac{d \phi}{2 \pi} \exp \left[-i Q \phi+z\left(\lambda_{+} e^{i \phi}+\lambda_{-} e^{-i \phi}\right)+\widetilde{z}\left(\widetilde{\lambda}_{+} e^{i 2 \phi}+\widetilde{\lambda}_{-} e^{-i 2 \phi}\right)\right]=\sum_{k=-\infty}^{\infty} I_{Q-2 k}(2 z) I_{k}(2 \widetilde{z}),
\end{aligned}
$$


where we have used the relation $\exp \left[x\left(t+\frac{1}{t}\right)\right]=\sum_{k=-\infty}^{\infty} t^{k} I_{k}(2 x)$. The $z$ and $\tilde{z}$ in Eq. (46) are the one-particle partition functions for charges \pm 1 and \pm 2 , respectively. In terms of variables $c_{m \pm}(m=1,2,4)$

$$
c_{m \pm}=\frac{\sum_{k=-\infty}^{\infty} I_{Q \mp m-2 k}(2 z) I_{k}(2 \widetilde{z})}{\sum_{k=-\infty}^{\infty} I_{Q-2 k}(2 z) I_{k}(2 \widetilde{z})}
$$

one finds:

$$
\begin{array}{ll}
\left\langle N_{ \pm}\right\rangle_{\text {c.e. }}=c_{1 \pm} z, & \left\langle\widetilde{N}_{ \pm}\right\rangle_{\text {c.e. }}=c_{2 \pm} \widetilde{z} \\
\left\langle N_{ \pm}^{2}\right\rangle_{\text {c.e. }}=c_{1 \pm} z+c_{2 \pm} z^{2}, & \left\langle\widetilde{N}_{ \pm}^{2}\right\rangle_{c . e .}=c_{2 \pm} \widetilde{z}+c_{4 \pm} \widetilde{z}^{2} .
\end{array}
$$

From Eqs. (48 49) it follows:

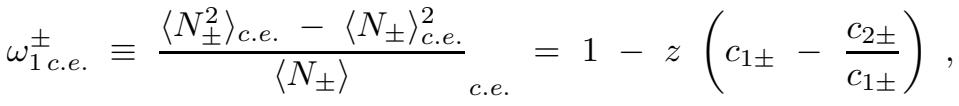

$$
\begin{aligned}
& \omega_{2 \text { c.e. }}^{ \pm} \equiv \frac{\left\langle\widetilde{N}_{ \pm}^{2}\right\rangle_{c . e .}-\left\langle\widetilde{N}_{ \pm}\right\rangle_{c . e .}^{2}}{\left\langle\widetilde{N}_{ \pm}\right\rangle_{c . e .}}=1-\widetilde{z}\left(c_{2 \pm}-\frac{c_{4 \pm}}{c_{2 \pm}}\right) \text {, } \\
& \omega_{c . e .}^{ \pm} \equiv \frac{\left\langle\left(N_{ \pm}+\widetilde{N}_{ \pm}\right)^{2}\right\rangle_{c . e .}-\left\langle N_{ \pm}+\widetilde{N}_{ \pm}\right\rangle_{c . e .}^{2}}{\left\langle N_{ \pm}+\widetilde{N}_{ \pm}\right\rangle_{c . e .}}=1+\frac{z^{2} c_{2 \pm}+\widetilde{z}^{2} c_{4 \pm}+2 z \widetilde{z} c_{3 \pm}}{z c_{1 \pm}+\widetilde{z} c_{2 \pm}}-\left(z c_{1 \pm}+\widetilde{z} c_{2 \pm}\right) .
\end{aligned}
$$

To illustrate the specific features of the considered system we present the CE results in the case $Q=0$. As always, all $\omega$ 's are equal to 1 in the GCE. For $\langle Q\rangle_{\text {g.c.e. }}=0$ one also has $\left\langle N_{ \pm}\right\rangle_{\text {g.c.e. }}=z$ and $\left\langle\widetilde{N}_{ \pm}\right\rangle_{\text {g.c.e. }}=\widetilde{z}$. To calculate Eqs. (48) 52) for finite values of $z$ and $\widetilde{z}$ one can effectively use Eq. (47). In the thermodynamic limit $V \rightarrow \infty$ both $z \rightarrow \infty$ and $\widetilde{z} \rightarrow \infty$. In this case it is convenient to return to the integral over $\phi$ representation in Eq. (46) and use it also for the derivatives of the CE partition function with respect to $\lambda_{ \pm}$and $\widetilde{\lambda}_{ \pm}$. Using the saddle point method to calculate the $\phi$-integrals one finds then for $z, \widetilde{z} \gg 1$ :

$$
\begin{aligned}
\left\langle N_{ \pm}\right\rangle_{c . e .} & \simeq z\left[1-\frac{1}{4(z+4 \widetilde{z})}\right],\left\langle\widetilde{N}_{ \pm}\right\rangle_{c . e .}=\widetilde{z}\left(1-\frac{1}{z+4 \widetilde{z}}\right) \\
\omega_{1 c . e .}^{ \pm} & \simeq 1-\frac{z}{2(z+4 \widetilde{z})}, \quad \omega_{2 c . e .}^{ \pm} \simeq 1-\frac{2 \widetilde{z}}{z+4 \widetilde{z}}, \quad \omega_{c . e .}^{ \pm} \simeq 1-\frac{(z+2 \widetilde{z})^{2}}{2(z+\widetilde{z})(z+4 \widetilde{z})} .
\end{aligned}
$$

From the above formulas one finds that $\omega_{c . e .}^{ \pm} \simeq 0.5$ if $\widetilde{z} / z$ is either much smaller or much larger than 1 . The scaled variance $\omega_{c . e \text {. }}^{ \pm}$has a maximum at $z=2 \widetilde{z}$. At this point one finds $\omega_{c . e .}^{ \pm}=5 / 9, \omega_{1 \text { c.e. }}^{ \pm}=5 / 6$ and $\omega_{2 \text { c.e. }}^{ \pm}=2 / 3$.

\section{QUANTUM STATISTICS EFFECTS}

It is instructive to apply a different technique 7] to calculate the fluctuations of the thermodynamical quantities with the exact conservation laws imposed. This method allows to find the values of the CE fluctuations in the thermodynamic limit and include the effects of quantum statistics.

The ideal quantum gas of the identical Bose or Fermi particles and antiparticles can be characterized by the occupation numbers $n_{p}^{ \pm}$of the one-particle states labeled by momenta p. The GCE average values and fluctuations are [1]]:

$$
\begin{aligned}
\left\langle n_{p}^{ \pm}\right\rangle_{\text {g.c.e. }} & =\frac{1}{\exp \left[\left(\sqrt{p^{2}+m^{2}} \mp \mu\right) / T\right]-\gamma}, \\
\left\langle\Delta n_{p}^{ \pm 2}\right\rangle_{\text {g.c.e. }} & \equiv\left\langle\left(n_{p}^{ \pm}\right)^{2}\right\rangle_{\text {g.c.e. }}-\left\langle n_{p}^{ \pm}\right\rangle_{\text {g.c.e. }}^{2}=\left\langle n_{p}^{ \pm}\right\rangle_{\text {g.c.e. }}\left(1+\gamma\left\langle n_{p}^{ \pm}\right\rangle_{\text {g.c.e. }}\right) \equiv v_{p}^{ \pm 2},
\end{aligned}
$$

where $\gamma$ is equal to +1 and -1 for Bose and Fermi statistics, respectively ( $\gamma=0$ corresponds to the Boltzmann approximation). These expressions are microscopic in a sense that they describe the average values and fluctuations of a single mode with momentum $p$. However, the average values of all macroscopic quantities of the system can be determined through the average occupation numbers of these single modes. The fluctuations of the macroscopic observables can be written in terms of the microscopic correlator $\left\langle\Delta n_{p}^{\alpha} \Delta n_{k}^{\beta}\right\rangle_{\text {g.c.e. }}$, where $\alpha, \beta$ are + and(or) -. This correlator can be presented as:

$$
\left\langle\Delta n_{p}^{\alpha} \Delta n_{k}^{\beta}\right\rangle_{\text {g.c.e. }}=v_{p}^{\alpha 2} \delta_{p k} \delta_{\alpha \beta}
$$


due to the statistical independence of different quantum levels and different charge states in the GCE. The variances of the total number of (negatively) positively charged particles $N_{\alpha}=\sum_{p} n_{p}^{\alpha}$ are equal to:

$$
\left\langle\Delta N_{\alpha}^{2}\right\rangle_{g . c . e .} \equiv\left\langle N_{\alpha}^{2}\right\rangle_{g . c . e .}-\left\langle N_{\alpha}\right\rangle_{g . c . e .}^{2}=\sum_{p, k}\left(\left\langle n_{p}^{\alpha} n_{k}^{\alpha}\right\rangle_{g . c . e .}-\left\langle n_{p}^{\alpha}\right\rangle_{g . c . e .}\left\langle n_{k}^{\alpha}\right\rangle_{g . c . e .}\right)=\sum_{p, k}\left\langle\Delta n_{p}^{\alpha} \Delta_{k}^{\alpha}\right\rangle_{g . c . e .}=\sum_{p} v_{p}^{\alpha 2} .
$$

We have assumed above that the quantum $p$-levels are non-degenerate. In fact each level should be further specified by the projection of a particle spin. Thus, each $p$-level splits into $g=2 j+1$ sub-levels. It will be assumed that the $p$-summation includes all these sub-levels too. The degeneracy factor enters explicitly when one substitutes, in the thermodynamic limit, the summation over discrete levels by the integration:

$$
\sum_{p} \ldots=\frac{g V}{2 \pi^{2}} \int_{0}^{\infty} p^{2} d p
$$

The scaled variance $\omega_{g . c . e .}^{\alpha}$ in the thermodynamical limit $V \rightarrow \infty$ reads:

$$
\omega_{\text {g.c.e. }}^{\alpha} \equiv \frac{\left\langle N_{\alpha}^{2}\right\rangle_{\text {g.c.e. }}-\left\langle N_{\alpha}\right\rangle_{\text {g.c.e. }}^{2}}{\left\langle N_{\alpha}\right\rangle_{\text {g.c.e. }}}=\frac{\sum_{p, k}\left\langle\Delta n_{p}^{\alpha} \Delta n_{k}^{\alpha}\right\rangle_{g . c . e .}}{\sum_{p}\left\langle n_{p}^{\alpha}\right\rangle_{\text {g.c.e. }}}=\frac{\sum_{p} v_{p}^{\alpha 2}}{\sum_{p}\left\langle n_{p}^{\alpha}\right\rangle_{\text {g.c.e. }}} \simeq \frac{\int_{0}^{\infty} p^{2} d p v_{p}^{\alpha 2}}{\int_{0}^{\infty} p^{2} d p\left\langle n_{p}^{\alpha}\right\rangle_{\text {g.c.e. }}} .
$$

The Eq. (58) corresponds to the particle number fluctuations in the GCE. To illustrate the role of quantum statistics let us consider the case of $\mu=0$, i.e. $\langle Q\rangle_{g . c . e .}=0$, where $Q \equiv \sum_{p, \alpha} q^{\alpha} n_{p}^{\alpha}$. In what follows we assume $q^{+}=1$ and $q^{-}=-1$, however, the formulas below are valid for any values of $q^{+}$and $q^{-}=-q^{+}$. From Eqs. (56) and (58) one finds $\omega_{\text {g.c.e. }}^{ \pm \text {Boltz }}=1$ in the Boltzmann limit $(\gamma=0), \omega_{\text {g.c.e. }}^{ \pm \text {Bose }}>1$ for the Bose particles $(\gamma=1)$ and $\omega_{\text {g.c.e. }}^{ \pm \overline{F e r m i}}<1$ for the Fermi particles $(\gamma=-1)$. The strongest quantum effects correspond to $m / T \rightarrow 0$ :

$$
\omega_{\text {g.c.e. }}^{ \pm \text {Boltz }}=1, \quad \omega_{\text {g.c.e. }}^{ \pm \text {Bose }}=\frac{\pi^{2}}{6 \zeta(3)} \simeq 1.368, \quad \omega_{\text {g.c.e. }}^{ \pm F e r m i}=\frac{\pi^{2}}{9 \zeta(3)} \simeq 0.912 .
$$

The scaled variance $\omega_{\text {g.c.e. }}^{\text {ch }}$ for all charged particles can be easily obtained from (58) by replacing $\sum_{p} \rightarrow \sum_{p \alpha}$, and one finds:

$$
\omega_{\text {g.c.e. }}^{\text {ch Boltz }}=\omega_{\text {g.c.e. }}^{ \pm \text {Boltz }}, \quad \omega_{\text {g.c.e. }}^{\text {ch Bose }}=\omega_{\text {g.c.e. }}^{ \pm \text {Bose }}, \quad \omega_{\text {g.c.e. }}^{\text {ch Fermi }}=\omega_{\text {g.c.e. }}^{ \pm F e r m i}
$$

The formula for the microscopic correlator is modified if we impose the exact charge conservation in our equilibrated system. For this purpose we introduce the equilibrium probability distribution $W\left(n_{p}^{\alpha}\right)$ of the occupation numbers. As a first step we assume that each $n_{p}^{\alpha}$ fluctuates independently according to the Gauss distribution law with the average value $\left\langle n_{p}^{\alpha}\right\rangle_{g . c . e . ~(55)}$ and the mean square deviation $v_{p}^{\alpha 2}$ (56):

$$
W\left(n_{p}^{\alpha}\right) \propto \prod_{p, \alpha} \exp \left[-\frac{\left(\Delta n_{p}^{\alpha}\right)^{2}}{2 v_{p}^{\alpha 2}}\right]
$$

To justify this assumption (see Ref. [7]) one can consider the sum of $n_{p}^{\alpha}$ in small momentum volume $(\Delta p)^{3}$ with the center at $p$. At fixed $(\Delta p)^{3}$ and $V \rightarrow \infty$ the average number of particles inside $(\Delta p)^{3}$ becomes large. Each particle configuration inside $(\Delta p)^{3}$ consists of $(\Delta p)^{3} \cdot V /(2 \pi)^{3} \gg 1$ statistically independent terms, each with the average value $\left\langle n_{p}^{\alpha}\right\rangle_{\text {g.c.e. (55) }}$ and the scaled variance $v_{p}^{\alpha 2}$ (56). From the central limit theorem it then follows that the probability distribution for the fluctuations inside $(\Delta p)^{3}$ should be Gaussian. In fact, we always convolve $n_{p}^{\alpha}$ with some smooth function of $p$, so instead of writing the Gaussian distribution for the sum of $n_{p}^{\alpha}$ in $(\Delta p)^{3}$ we can use it directly for $n_{p}^{\alpha}$.

The average value of the conserved charge $Q=\sum_{p, \alpha} q^{\alpha} n_{p}^{\alpha}$ is regulated in the GCE by the chemical potential $\mu$. If we impose an exact charge conservation, $\Delta Q=\sum_{p, \alpha} q^{\alpha} \Delta n_{p}^{\alpha}=0$, the distribution (61) will be modified as:

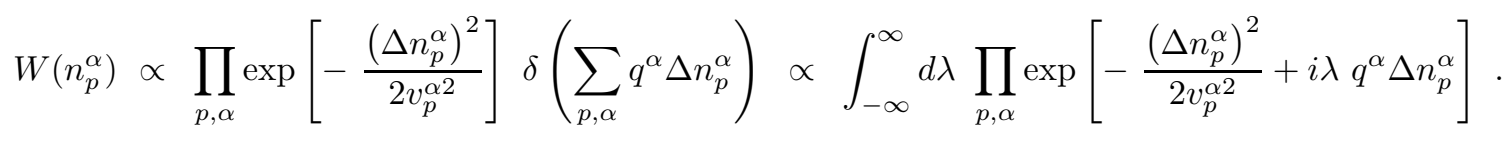

It is convenient to generalize distribution (62) to $W\left(n_{p}^{\alpha}, \lambda\right)$ using further the integration along imaginary axis in the $\lambda$-plane. After completing squares one gets:

$$
W\left(n_{p}^{\alpha}, \lambda\right) \propto \prod_{p, \alpha} \exp \left[-\frac{\left(\Delta n_{p}^{\alpha}-\lambda v_{p}^{\alpha 2} q^{\alpha}\right)^{2}}{2 v_{p}^{\alpha 2}}+\frac{\lambda^{2}}{2} v_{p}^{\alpha 2} q^{\alpha 2}\right]
$$


and the average values are now calculated as:

$$
\langle\ldots\rangle=\frac{\int_{-i \infty}^{i \infty} d \lambda \int_{-\infty}^{\infty} \prod_{p, \alpha} d n_{p}^{\alpha} \ldots W\left(n_{p}^{\alpha}, \lambda\right)}{\int_{-i \infty}^{i \infty} d \lambda \int_{-\infty}^{\infty} \prod_{p, \alpha} d n_{p}^{\alpha} W\left(n_{p}^{\alpha}, \lambda\right)}
$$

The Eq. (64) has the meaning of the CE averaging in the thermodynamic limit $V \rightarrow \infty$. One easily finds

$$
\left\langle\left(\Delta n_{p}^{\alpha}-v_{p}^{\alpha 2} \lambda q^{\alpha}\right)\left(\Delta n_{k}^{\beta}-v_{k}^{\beta 2} \lambda q^{\beta}\right)\right\rangle=\delta_{p k} \delta_{\alpha \beta} v_{p}^{\alpha 2}, \quad\left\langle\lambda^{2}\right\rangle=-\left(\sum_{p, \alpha} v_{p}^{\alpha 2} q^{\alpha 2}\right)^{-1}, \quad\left\langle\left(\Delta n_{p}^{\alpha}-v_{p}^{\alpha 2} \lambda q^{\alpha}\right) \lambda\right\rangle=0
$$

so that it follows:

$$
\begin{aligned}
\left\langle\Delta n_{p}^{\alpha} \Delta n_{k}^{\beta}\right\rangle & =\delta_{p k} \delta_{\alpha \beta} v_{p}^{\alpha 2}-v_{p}^{\alpha 2} q^{\alpha} v_{k}^{\beta 2} q^{\beta}\left\langle\lambda^{2}\right\rangle+\left\langle\Delta n_{p}^{\alpha} \lambda\right\rangle v_{k}^{\beta 2} q^{\beta}+\left\langle\Delta n_{k}^{\beta} \lambda\right\rangle v_{p}^{\alpha 2} q^{\alpha} \\
& =\delta_{p k} \delta_{\alpha \beta} v_{p}^{\alpha 2}+v_{p}^{\alpha 2} q^{\alpha} v_{k}^{\beta 2} q^{\beta}\left\langle\lambda^{2}\right\rangle=\delta_{p k} \delta_{\alpha \beta} v_{p}^{\alpha 2}-\frac{v_{p}^{\alpha 2} q^{\alpha} v_{k}^{\beta 2} q^{\beta}}{\sum_{p, \alpha} v_{p}^{\alpha 2} q^{\alpha 2}}
\end{aligned}
$$

By means of Eq. [65] we obtain:

$$
\omega_{c . e .}^{\alpha} \equiv \frac{\left\langle N_{\alpha}^{2}\right\rangle-\left\langle N_{\alpha}\right\rangle^{2}}{\left\langle N_{\alpha}\right\rangle}=\frac{\sum_{p} v_{p}^{\alpha 2}}{\sum_{p}\left\langle n_{p}^{\alpha}\right\rangle_{g . c . e .}}-\frac{\left(\sum_{p} v_{p}^{\alpha 2} q^{\alpha}\right)^{2}}{\sum_{p}\left\langle n_{p}^{\alpha}\right\rangle_{g . c . e .} \sum_{p, \alpha} v_{p}^{\alpha 2} q^{\alpha 2}}
$$

The Eq. (55) leads to $v_{p}^{\alpha 2}=\left\langle n_{p}^{\alpha}\right\rangle_{\text {g.c.e. }}$ in the Boltzmann approximation, so that from Eq. (66) one finds $(y \equiv Q / 2 z=$ $\sinh (\mu / T))$ :

$$
\omega_{c . e .}^{\alpha}=1-\frac{\exp (\alpha \mu / T)}{\exp (\mu / T)+\exp (-\mu / T)}=\frac{1}{2}-\alpha \frac{y}{2 \sqrt{1+y^{2}}},
$$

which coincides with Eq. (38). Formula $\omega_{c . e .}^{c h}$ can be obtained from (66) after replacing $\sum_{p} \rightarrow \sum_{p, \alpha}$, and it is the same as Eq. (39). At $\mu=0$ from Eq. (66) we find the CE scaled variances:

$$
\begin{aligned}
& \omega_{\text {c.e. }}^{ \pm \text {Boltz }}=\frac{1}{2}, \\
& \omega_{\text {c.e. }}^{ \pm \text {Bose }}=\frac{\pi^{2}}{12 \zeta(3)} \simeq 0.684, \\
& \omega_{\text {c.e. }}^{ \pm F e r m i}=\frac{\pi^{2}}{18 \zeta(3)} \simeq 0.456, \\
& \omega_{\text {c.e. }}^{\text {ch Boltz }}=2 \omega_{\text {g.c.e. }}^{ \pm \text {Boltz }}, \\
& \omega_{\text {c.e. }}^{\text {ch Bose }}=2 \omega_{\text {c.e. }}^{ \pm \text {Bose }} \text {, } \\
& \omega_{\text {c.e. }}^{\text {ch Fermi }}=2 \omega_{\text {c.e. }}^{ \pm \text {Fermi }} \text {. }
\end{aligned}
$$

As seen from Eqs. (5968) the scaled variance of (negative) positive particles with Bose or Fermi statistics in the $\mathrm{CE}$ is as half as large as the corresponding scaled variances in the GCE. Therefore, the CE suppression of the particle number fluctuations in the thermodynamic limit works at $\mu=0$ in the quantum systems similar to that in the Boltzmann case. This result can be rephrased in another way: the Bose enhancement and Fermi suppression of the GCE fluctuations remain the same in the CE for the $\omega_{c . e}^{ \pm}$at $\mu=0$ in the thermodynamic limit. The Eq. (69) demonstrates that the scaled variances of all charged particles in the CE for any statistics are by a factor of 2 larger than the corresponding scaled variances for (negative) positive particles, whereas in the GCE these scaled variances presented by Eq. (60) are equal to each other.

Comparing Eq. (65) and Eq. (57) one notices the changes of the microscopic correlator due to an exact charge conservation. Namely, in the CE the fluctuations of each mode is reduced, i.e. the $\left\langle\left(\Delta n_{p}^{\alpha}\right)^{2}\right\rangle$ calculated from Eq. (65) is smaller than that in Eq. (56), and the anticorrelations between different modes $p \neq k$ and the same charge states $\alpha=\beta$ appear. These two changes of the microscopic correlator result in a suppression of the CE scaled variances $\omega_{c . e}^{\alpha}$.

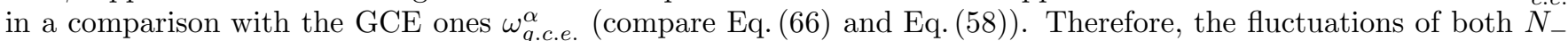
and $N_{+}$are always suppressed in the CE. As we have seen in the previous sections the behavior of $N_{c h}$ fluctuations in the CE can be more complicated. This is because of the correlations of different modes $p \neq k$ for the different charge states $\alpha=-\beta$ (i.e. the second term in the right hand side of Eq. (65) is positive for $\alpha=-\beta$ ).

The exact charge conservation should also lead to the canonical suppression of $\left\langle n_{p}^{\alpha}\right\rangle$, and this should result in the canonical suppression effects for $\left\langle N_{\alpha}\right\rangle$. They are, however, absent in the present formulation, so that formula (65) for the microscopic correlator is not enough to calculate $\left\langle N_{\alpha}^{2}\right\rangle$ and $\left\langle N_{\alpha}\right\rangle^{2}$ separately with an accuracy corresponding to the effects of the canonical suppression. Nevertheless, it does allow us to calculate their difference $\left\langle\left(\Delta N_{\alpha}\right)^{2}\right\rangle$ with the effects of the CE correctly included. This means that the canonical suppression effects in the occupation 
numbers $\left\langle n_{p}^{\alpha}\right\rangle$ lead to the changes of the order of $\left\langle N_{\alpha}\right\rangle$ in both $\left\langle N_{\alpha}^{2}\right\rangle$ and $\left\langle N_{\alpha}\right\rangle^{2}$, but these changes are the same and the correction terms are cancelled in the calculation of $\left\langle\left(\Delta N_{\alpha}\right)^{2}\right\rangle$. Therefore, the macroscopic fluctuations of multiplicities are not affected by the CE corrections to the average particle numbers. The scaled variances of the CE in the thermodynamic limit $V \rightarrow \infty$ feel the consequences of an exact charge conservation due to the suppression of the single mode fluctuations $\left\langle\left(\Delta n_{p}^{\alpha}\right)^{2}\right\rangle$ and due to the (anticorrelations ) correlations between different modes $p \neq k$ with the (same) different charge states $\alpha, \beta$. All these effects are absent in the GCE.

\section{A SYSTEM WITH TWO CONSERVED CHARGES}

In the previous sections we have considered the system with one conserved charge. In high energy collisions the measurements of fluctuations for the particle numbers and (transverse) energies are mainly done for electrically charged hadrons. Therefore, in applications of the CE results to an analysis of the data on fluctuations it would be reasonable to start with the case when the charge $Q$ is assumed to be an electric charge. On the other hand, other conserved charges are also present in the system created in high energy collisions. In this section we consider the system with two exactly conserved charges - electric charge $Q$ and baryonic number $B$. As an example we study the ideal pion-nucleon gas and neglect the quantum statistics effects. This is the simplest realistic case where we can study the influence of an exact $B$ conservation to the CE fluctuations of the electrically charged particles. The partition function of this system in the $\mathrm{CE}$ is:

$$
\begin{aligned}
& Z_{\text {c.e. }}(V, T, Q, B)=\sum_{N_{p}, N_{\bar{p}}=0}^{\infty} \sum_{N_{n}, N_{\bar{n}}=0}^{\infty} \sum_{N_{\pi}, N_{\bar{\pi}}=0}^{\infty} \frac{\left(\lambda_{p} z_{p}\right)^{N_{p}}}{N_{p} !} \frac{\left(\lambda_{\bar{p}} z_{\bar{p}}\right)^{N_{\bar{p}}}}{N_{\bar{p}} !} \frac{\left(\lambda_{n} z_{n}\right)^{N_{n}}}{N_{n} !} \frac{\left(\lambda_{\bar{n}} z_{\bar{n}}\right)^{N_{\bar{n}}}}{N_{\bar{n}} !} \frac{\left(\lambda_{\pi^{+}} z_{\pi}\right)^{N_{\pi^{+}}}}{N_{\pi^{+}} !} \frac{\left(\lambda_{\pi^{-}} z_{\pi}\right)^{N_{\pi}-}}{N_{\pi^{-}} !} \\
& \times \delta\left[\left(N_{p}-N_{\bar{p}}+N_{\pi^{+}}-N_{\pi^{-}}\right)-Q\right] \delta\left[\left(N_{p}-N_{\bar{p}}+N_{n}-N_{\bar{n}}\right)-B\right] \\
& =\int_{0}^{2 \pi} \frac{d \varphi}{2 \pi} \int_{0}^{2 \pi} \frac{d \phi}{2 \pi} \exp (-i Q \varphi-i B \phi) \times \exp \left[z_{p}\left(\lambda_{p} e^{i(\varphi+\phi)}+\lambda_{\bar{p}} e^{-i(\varphi+\phi)}\right)\right] \\
& \times \exp \left[z_{n}\left(\lambda_{n} e^{i \phi}+\lambda_{\bar{n}} e^{-i \phi}\right)\right] \times \exp \left[z_{\pi}\left(\lambda_{\pi^{+}} e^{i \varphi}+\lambda_{\pi^{-}} e^{-i \varphi}\right)\right]=\sum_{k=-\infty}^{\infty} I_{k-Q}\left(2 z_{p}\right) I_{k+B-Q}\left(2 z_{n}\right) I_{k}\left(2 z_{\pi}\right), \quad(70)
\end{aligned}
$$

where we have used that $\exp [x(t+1 / t)]=\sum_{k=0}^{\infty} t^{k} I_{k}(2 x)$. From Eq. (70) it follows:

$$
\left\langle N_{j, \alpha}\right\rangle_{c . e .}=c_{1, \alpha}^{j} z_{j}, \quad\left\langle N_{j, \alpha}^{2}\right\rangle_{c . e .}=c_{1, \alpha}^{j} z_{j}+c_{2, \alpha}^{j} z_{j}^{2},
$$

where $j$ numerates pion, neutron and proton, $\alpha=1$ corresponds to particles $\pi^{+}, n, p$ and $\alpha=-1$ to antiparticles $\pi^{-}, \bar{n}, \bar{p}$, and $(m=1,2)$

$$
\begin{aligned}
c_{m, \alpha}^{p} & =\sum_{k=-\infty}^{\infty} I_{k+\alpha \cdot m-Q}\left(2 z_{p}\right) I_{k+B-Q}\left(2 z_{n}\right) I_{k}\left(2 z_{\pi}\right) \times\left[Z_{c . e .}(V, T, Q, B)\right]^{-1}, \\
c_{m, \alpha}^{n} & =\sum_{k=-\infty}^{\infty} I_{k-Q}\left(2 z_{p}\right) I_{k+\alpha \cdot m+B-Q}\left(2 z_{n}\right) I_{k}\left(2 z_{\pi}\right) \times\left[Z_{c . e .}(V, T, Q, B)\right]^{-1}, \\
c_{m, \alpha}^{\pi} & =\sum_{k=-\infty}^{\infty} I_{k+\alpha \cdot m-Q}\left(2 z_{p}\right) I_{k+\alpha \cdot m+B-Q}\left(2 z_{n}\right) I_{k}\left(2 z_{\pi}\right) \times\left[Z_{c . e .}(V, T, Q, B)\right]^{-1} .
\end{aligned}
$$

Formulas for the cross-averages $\left\langle N_{i} N_{j}\right\rangle$ can be obtained in the similar manner. The calculations with Eqs. (72 74) are effective for small systems. In this case the $k$-sums in the above equations converge rapidly and small number of terms lead to the accurate results. In the limit of large system volume we can use another technique, similar to that developed in the previous section. This leads to simple analytical results. Using this method one can obtain, for example, the scaled variances for (negatively) positively charged particles in the thermodynamic limit. The same pictures can be obtained directly from Eqs. (72 74) by numerical calculations at $z_{p}, z_{n}, z_{\pi} \gg 1$.

First, we consider the case when the electric charge $Q$ is exactly conserved and the baryonic number $B$ conservation is treated within the GCE. This results in:

$$
\omega_{Q}^{ \pm}=1-\frac{z_{p}^{ \pm}+z_{\pi}^{ \pm}}{z_{p} x_{p}+z_{\pi} x_{\pi}},
$$


where

$$
z_{j}^{ \pm}=z_{j} \exp \left( \pm \frac{\mu_{j}}{T}\right), \quad x_{j}=\exp \left(\frac{\mu_{j}}{T}\right)+\exp \left(-\frac{\mu_{j}}{T}\right),
$$

and the chemical potentials $\mu_{j}$ are equal to $\mu_{p}=\mu_{Q}+\mu_{B}$ for protons and $\mu_{\pi}=\mu_{Q}$ for $\pi^{+}$-mesons. When both $Q$ and $B$ are exactly conserved, the CE scaled variances of (negatively) positively charged particles are equal to:

$$
\omega_{Q, B}^{ \pm}=1-\frac{z_{p}^{ \pm 2}\left(z_{n} x_{n}+z_{\pi} x_{\pi}\right)+z_{\pi}^{ \pm 2}\left(z_{p} x_{p}+z_{n} x_{n}\right)+2 z_{p}^{ \pm} z_{\pi}^{ \pm} z_{n} x_{n}}{\left(z_{p}^{ \pm}+z_{\pi}^{ \pm}\right)\left(z_{p} x_{p} z_{n} x_{n}+z_{p} x_{p} z_{\pi} x_{\pi}+z_{n} x_{n} z_{\pi} x_{\pi}\right)},
$$

where $x_{n}=\exp \left(\mu_{B} / T\right)+\exp \left(-\mu_{B} / T\right)$. Let us repeat that both $\omega_{Q}^{ \pm}$(75) and $\omega_{Q, B}^{ \pm}$(77) are obtained in the thermodynamic limit $V \rightarrow \infty$. The $\omega_{Q}^{ \pm}$(75) corresponds to the CE for electric charge and the GCE for baryonic number. The $\omega_{Q, B}^{ \pm}$(77) corresponds the CE for both conserved charges. It is easy to prove that $\omega_{Q, B}^{ \pm} \leq \omega_{Q}^{ \pm}$, i.e. an additional exact conservation law reduces the fluctuations. However, the additional CE suppression of the scaled variances of (negatively) positively charged particles due to the exact baryonic number conservation is rather small. We have plotted (75) and (77) in Fig.6 for $\mu_{Q}=0$ to study the influence of baryon charge conservation on the fluctuations of electrically charged particles. As one can see from Fig. 6 the exact CE baryonic charge conservation leads to a little

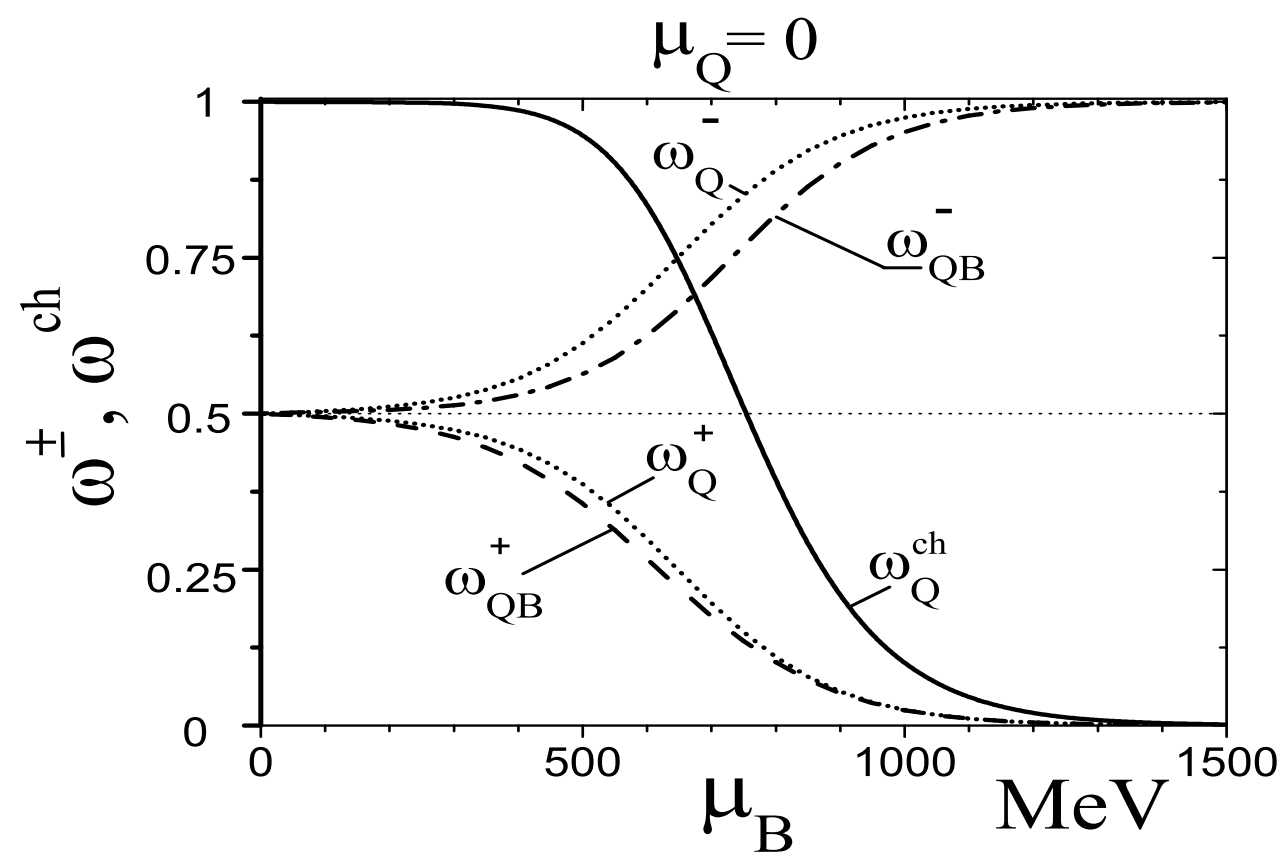

FIG. 6: The scaled variances $\omega_{Q, B}^{+}$(dashed line) and $\omega_{Q, B}^{-}$(dashed-dotted line) given by Eq. (77). The dotted lines show the scaled variances $\omega_{Q}^{+}$and $\omega_{Q}^{-}$given by Eq. (75). The solid line presents the scaled variance for all charged particles $\omega_{Q}^{c h}$. The results correspond to $T=120 \mathrm{MeV}$.

additional suppression and does not change the result $\omega^{+}=\omega^{-}=0.5$ for zero values of the baryonic and electric net charges. Moreover, one can prove that at zero net charges any ideal Boltzmann gas with two exactly conserved charges (i.e. for any combination of particle charges and their masses) leads to the scaled variances equal to $\omega^{ \pm}=0.5$ in the thermodynamic limit.

On the other hand, Fig. 6 demonstrates a strong dependence of the $\omega_{Q}^{+}$and $\omega_{Q}^{-}$values on the net baryonic density, it is not important whether the baryonic number treated within the $\mathrm{CE}$ or the GCE. The matter is that in the pionnucleon gas the electric charge equals to $Q=N_{p}-N_{\bar{p}}+N_{\pi^{+}}-N_{\pi^{-}}$. At $\mu_{B} \simeq 0$ the electric charge of the system is close to zero. Then one finds $\omega_{Q}^{+} \simeq \omega_{Q}^{-} \simeq 0.5$ (compare to Fig. 4 at $y=0.1$ ). The $\mu_{B}>0$ leads to $\left\langle N_{p}\right\rangle>\left\langle N_{\bar{p}}\right\rangle$, and this means a non-zero electric charge of the system. In this case an exact electric charge conservation leads to $\omega_{Q}^{-}>\omega_{Q}^{+}$(see Fig. 4). At $\mu_{B} \gg T$ the electric charge density becomes large due to $\left\langle N_{p}\right\rangle /\left\langle N_{\bar{p}}\right\rangle \gg 1$, so that $\omega_{Q}^{+} \rightarrow 0$ and $\omega_{Q}^{-} \rightarrow 1$ (compare to Fig. 4 at $y=2$ ). 


\section{SUMMARY AND CONCLUSIONS}

We have considered the particle number and energy fluctuations for different systems within the canonical ensemble formulation. The results are compared to those in the grand canonical ensemble. We have studied the system with arbitrary number of different particle species and non-zero net charge in Secs. II and III. An exact charge conservation reduces the values of $N_{+}$and $N_{-}$fluctuations in the thermodynamic limit. At the non-zero net charge $Q$ the canonical ensemble predicts a difference for the fluctuations of $N_{+}$and $N_{-}$, they also different from the fluctuations of all charged particles $N_{c h}=N_{+}+N_{-}$. All these features of the canonical ensemble are in a striking difference with those in the grand canonical ensemble. We have demonstrated in Sec. IV that the energy fluctuations of the system are mainly determined by the fluctuations of the number of particles and have the same volume dependence. Therefore, the energy fluctuations are rather different in the canonical and grand canonical ensembles. We extend our canonical ensemble results and calculate the particle number fluctuations in the system of single and double charged particles in Sec. V, include the quantum statistics effects in Sec. VI, study the systems with two conserved charges in Sec. VII.

The canonical ensemble suppression effects for the charged particle multiplicities are well known, and they are successfully applied to the statistical description of hadron production in high energy collisions [3]. The canonical ensemble formulation explains, for example, the suppression in a production of strange hadrons and antibaryons in small systems, i.e. when the total numbers of strange particles or antibaryons are small. This consideration demonstrates a difference of the canonical and grand canonical ensembles - the statistical ensembles are not equivalent for small systems. When the size of the system increases all average quantities in both ensembles become equal. It means that in the thermodynamic limit $V \rightarrow \infty$ the canonical ensemble and grand canonical ensemble are equivalent. Results of Ref. [8] and the present study demonstrate that there are also the canonical ensemble effects for the fluctuations. In contrast to the canonical suppression of average multiplicities, the canonical effects for the multiplicity fluctuations do survive at $V \rightarrow \infty$ and they are even most clearly seen in the thermodynamic limit. The changes of the scaled variances due to an exact charge conservation of the canonical ensemble are not small (about 50 percent effects) and they are in general different for positively, negatively and all charged particles. To observe these new canonical ensemble effects in an analysis of the data on multiparticle production, several points should be clarified. To use the condition of an exact charge conservation one has to apply it to the system of all secondary hadrons formed in high energy collisions, and this should be done on the event-by-event basis as we are interested in the system fluctuations. In the experimental study only a fraction of produced particles with the conserved charges is detected. Introducing a probability $q$ that a single particle is accepted in the detector a simple relation between the scaled variance of the accepted particles, $\omega_{a c c}$, and the scaled variance of all particles in the statistical ensemble, $\omega$, was obtained [8]: $\omega_{a c c}=q \cdot \omega+(1-q)$. To observe the real event-byevent fluctuations $\omega$ one needs $q \simeq 1$, otherwise if $q \ll 1$ one always obtains $\omega_{a c c} \simeq 1$ and makes a wrong conclusion that the fluctuations correspond to the Poisson distribution. This fact is of a very general origin, and because of relatively small experimental acceptance a large part of the event-by-event fluctuations in high energy multiparticle production is lost. To observe many interesting event-by-event fluctuations, for example, due to the QCD critical point (see, e.g. [12] and references therein), one should accept an essential part of all secondary particles. In this case the role of an exact charge conservation would increase. It would also have a strong influence on an extraction of the so called dynamical fluctuations (see Ref. [6]) from the event-by-event data.

\section{Acknowledgments}

We would like to thank F. Becattini, A.I. Bugrij, T. Csörgő, M. Gaździcki, A. Keränen, A.P. Kostyuk, I.N. Mishustin, St. Mrówczyński, L.M. Satarov and Y.M. Sinyukov for useful discussions. We thank A. Swaving for help in the preparation of the manuscript. The work was supported by US Civilian Research and Development Foundation (CRDF) Cooperative Grants Program, Project Agreement UKP1-2613-KV-04.

[1] J. Cleymans, H. Satz, Z. Phys. C 57 (1993) 135; J. Sollfrank, M. Gaździcki, U. Heinz, J. Rafelski, ibid. 61 (1994) 659; G.D. Yen, M.I. Gorenstein, W. Greiner, S.N. Yang, Phys. Rev. C 56 (1997) 2210; F. Becattini, M. Gaździcki, J. Solfrank, Eur. Phys. J. C 5 (1998) 143; G.D. Yen, M.I. Gorenstein, Phys. Rev. C 59 (1999) 2788; P. Braun-Munzinger, I. Heppe, J. Stachel, Phys. Lett. B 465 (1999) 15; P. Braun-Munzinger, D. Magestro, K. Redlich, J. Stachel, ibid. 518 (2001) 41; F. Becattini, M. Gaździcki, A. Keranen, J. Mannienen, R. Stock, Phys. Rev. C 69 (2004) 024905.

[2] P. Braun-Munzinger, K. Redlich, J. Stachel, nucl-th/0304013 Review for Quark Gluon Plasma 3, eds. R.C. Hwa and X.-N. Wang, World Scientific,Singapore. 
[3] K. Redlich, L. Turko, Z. Phys. C 5 (1980) 541; J. Rafelski, M. Danos, Phys. Lett. B 97 (1980) 279. J. Cleymans, K Redlich, E Suhonen, Z. Phys. C 51 (1991) 137; J. Cleymans, A. Keränen, M. Marais, E. Suhonen, Phys. Rev. C 56 (1997) 2747; F. Becattini, Z. Phys. C 69 (1996) 485; F. Becattini, U. Heinz, ibid. 76 (1997) 269; J. Cleymans, H. Oeschler, K. Redlich, Phys. Rev. C 59 (1999) 1663; Phys. Lett. B 485 (2001) 27; M.I. Gorenstein, M. Gaździcki, W. Greiner, ibid. 483 (2000) 60; M.I. Gorenstein, A.P. Kostyuk, H. Stöcker, W. Greiner, ibid 509 (2001) 277.

[4] K. Werener, J. Aichelin, Phys. Rev. C 52 (1995) 1584; F. Liu, K. Werner, J. Aichelin, ibid 68 (2003) 024905; F. Liu, K. Werner, J. Aichelin, M. Bleicher, H. Stöcker, J. Phys. G 30 (2004) S589; F. Becattini, L. Ferroni, Eur. Phys. J. C 35 (2004) 243; hep-ph/0407117

[5] M.A. Stephanov, K. Rajagopal, E.V. Shuryak, Phys. Rev. Lett. 81 (1998) 4816; H. Heiselberg, Phys. Rep. 351 (2001) 161; S. Jeon, V. Koch, hep-ph/0304012, Review for Quark-Gluon Plasma 3, eds. R.C. Hwa and X.-N. Wang, World Scientific, Singapore.

[6] M. Gaździcki, M.I. Gorenstein, St. Mrówczyński, Phys. Lett. B 585 (2004) 115; M.I. Gorenstein, M. Gaździcki, O.S. Zozulya, ibid. 585 (2004) 237.

[7] M.A. Stephanov, K. Rajagopal, E.V. Shuryak, Phys. Rev. D 60 (1999) 114028.

[8] V.V. Begun, M. Gaździcki, M.I. Gorenstein, O.S. Zozulya, Phys. Rev. C 70(2004) 034901.

[9] V.V. Begun, M.I. Gorenstein, A.P. Kostyuk, O.S. Zozulya, nucl-th/0410044

[10] M. Abramowitz and I.E. Stegun, Handbook of Mathematical Functions (Dover, New York, 1964).

[11] L.D. Landau, E.M. Lifschitz, Statistical Physics (Fizmatlit, Moscow, 2001).

[12] M. Stephanov, hep-ph/0402115 


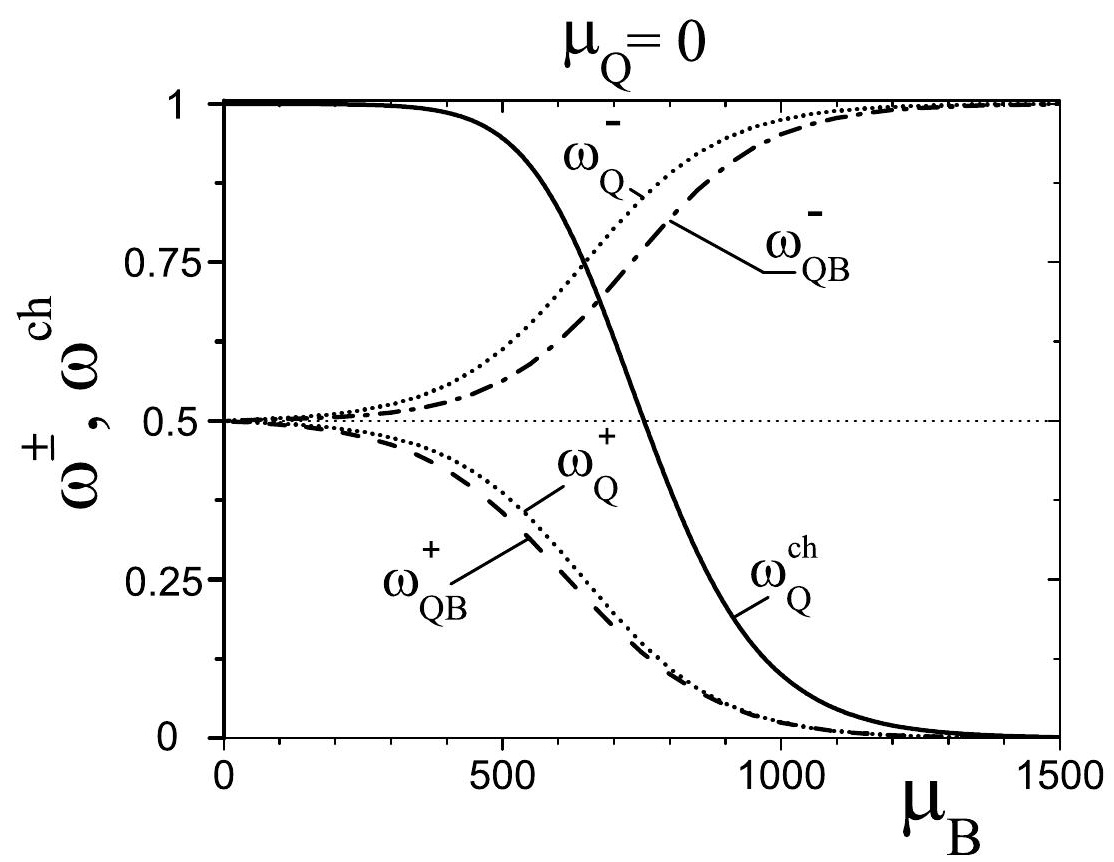

